\title{
Mesures thermiques des flux de sève et comportement hydrique des arbres. II. Évolution dans le temps des flux de sève et comportement hydrique des arbres en présence ou non d'une irrigation localisée.
}

\author{
B Cabibel, F Do
}

avec la participation technique de $\mathrm{J}$ Horoyan

INRA, station de science du sol, domaine Saint-Paul, BP 91, 84143 Montfavet cedex, France

(Reçu le 26 février 1990; accepté le 14 août 1991)

\begin{abstract}
Résumé - La mesure thermique des flux de sève dans les troncs et les racines de pommiers est utilisée pour caractériser les comportements relatifs d'arbres soumis ou non à une irrigation localisée. On estime leurs transpirations horaires et journalières et appréhende le fonctionnement hydrique des diverses composantes de leur systèmes racinaires. L'étude des cinétiques des flux, mesurées dans les troncs, permet de mettre en évidence l'apparition, dans le temps, d'un stress hydrique dans les arbres et donc leur besoin d'irrigation. L'analyse des cinétiques des flux racinaires élémentaires permet quant à elle d'appréhender et de quantifier l'hétérogénéité spatio-temporelle des fonctions puits racinaires pour l'eau.
\end{abstract}

Malus $=$ pommier $/$ tronc $/$ racine $/$ flux de sève $/$ mesure thermique $/$ irrigation localisée

Summary - Thermal measurement of sap flow and hydric behavior of trees. Il. Sap flow evolution and hydric behavior of irrigated and non-irrigated trees under trickle irrigation. A thermal method for measuring sap flow in apple tree trunks and roots has been applied for characterising relative behavior of non-irrigated trees and trees irrigated by trickle irrigation (fig 1). The hourly and daily transpiration rate was estimated (figs 2, 3 and 7; table I) and the soil water uptake of the different components of the root systems was determined (figs 5 and 6). In the trunks, the study of flow kinetics allowed characterization of hydric stress in trees and therefore the irrigation requirements of the orchard; and in the roots (fig 4), determination and estimation of the spatial and temporal heterogeneity of root sink function for soil-water.

Malus = apple tree $/$ trunk $/$ root $/$ sap flow $/$ thermal measurement $/$ trickle irrigation

\section{INTRODUCTION}

La fonction puits racinaire des arbres pour l'eau et son hétérogénéité spatio-temporelle sont, pour une demande climatique donnée, fonction de la disponibilité de l'eau du sol et de son évolution dans le temps. Son intensité globale peut être estimée par la mesure thermique des flux de sève traversant la surface conductrice du tronc, passage obligé de l'eau transpirée (Cohen et al, 1981; Granier, 1985; Valancogne et Nasr, 1989). L'analyse comparée, à moyen et long terme, des cinétiques des flux mesurés dans les arbres doit donc permettre, dans un système sol-planteatmosphère donné :

- de quantifier, en fonction de la disponibilité relative de l'eau du sol, les fonctions puits racinaires totales et leurs évolutions dans le temps,

- de caractériser l'état hydrique résultant des arbres, et de déterminer l'appartion éventuelle d'un stress hydrique et donc le besoin d'irrigation,

- d'appréhender l'hétérogénéité de l'absorption racinaire de l'eau dans le sol, par analyse des flux racinaires élémentaires dans chacune des racines de l'arbre. 
Dans ce qui suit, nous présentons des résultats caractérisant, au travers des variations temporelles des flux de sève mesurés simultanément dans les troncs et dans les racines :

- les comportements hydriques relatifs de pommiers soumis ou non à une irrigation localisée,

- l'hétérogénéité spatio-temporelle relative de leurs fonctions puits racinaires pour l'eau.

\section{MATÉRIEL ET MÉTHODES}

\section{Materiel}

\section{Le verger}

Planté en 1979, il est constitué de 4 rangs de 40 arbres, Reine des Reinettes greffées sur EM IX. Conduits en haie fruitière palissée, les arbres sont distants de $1,0 \mathrm{~m}$, les rangs de $3,5 \mathrm{~m}$. L'irrigation, mise en œuvre dès la plantation, est conduite par goutteurs, à raison d'un goutteur par arbre, placé à midistance de 2 arbres. La rampe d'irrigation est posée sur le sol le long de la raie de plantation. Les gout-

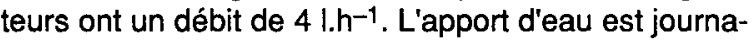
lier. II est déclenché à 10 h GMT. La dose d'eau est égale au jour $n$ à 0,8 ETP de celle du jour $n-1$. Pour le calcul des doses d'eau à apporter on ne comptabilise les précipitations que dans la limite de $30 \mathrm{~mm}$. On dispose d'arbres témoins non irrigués depuis la plantation. Le verger est constitué de 16 placettes élémentaires de 10 arbres réparties au hasard à l'implantation de l'irrigation (fig 1). Dans chaque traitement on étudie 4 arbres et, pour chaque arbre, 2 racines, dont les orientations sont respectivement parallèle et perpendiculaire à la ligne de plantation.

\section{Le climat du verger}

L'ETP horaire est calculée à l'aide d'un logiciel élaboré par le STEFCE de l'INRA-Montfavet. Les données climatiques nécessaires (températures sèche et humide, rayonnements, vitesse du vent, précipitations) sont mesurées, au sein même du verger, à l'aide d'une centrale météorologique automatique de type Cimel-Enerco 295-100 (Pinguet, 1984).

\section{La mesure des flux}

Elle est réalisée par voie thermique. Les flux sont directement quantifiés par le refroidissement qu'engendre une circulation de sève sur une sonde chauffée à puissance constante, une fraction de la chaleur étant alors dissipée par convection (Granier, 1985).
Les capteurs sont constitués de 2 thermocouples montés en opposition. Les sondes sont insérées radialement dans les troncs et les racines, au delà des assises corticales (Do, 1986).

On mesure en continu la variation des différences de température $\delta t$ entre la sonde chaude et une sonde identique non chauffante, sonde de référence, insérée en amont et de façon similaire dans le bois. Cette sonde de référence, supposée en équilibre thermique avec le milieu bois-sève, est censée mesurer la température au niveau de la sonde chaude s'il n'y avait pas de chauffage (fig 1).

Le flux de sève total $U$ est calculé à partir de la densité de flux $J u$ et de la surface de bois conductrice $S$ estimée dans le plan de la mesure :

$$
U=J u \cdot S
$$

La densité de flux $J u$ est déduite d'une relation d'étalonnage déterminée au laboratoire (Granier, 1985; Do, 1986), et la surface $S$ est soit directement estimée à partir de la mesure du rayon du bois conducteur par carottage direct dans les organes testés, soit déduite de relations statistiques liant cette surface au diamètre de l'organe étudié dans chaque traitement considéré.

La prise de mesure, dont le pas de temps peut varier de 1 à 60 min selon le phénomène étudié, est réalisée à l'aide d'un logiciel implanté sur compatible PC, qui pilote une acquisition de données SAM60-AOIP.

\begin{tabular}{|c|c|c|c|}
\hline Sec & $4 \mathrm{Lh}^{-1}$ & $2 \mathrm{Lh}^{-1}$ & $4 \mathrm{Lh}^{-1}$ \\
\hline $2 \mathrm{Lh}^{-1}$ & $4 \mathrm{Lh}^{-1}$ & $\mathrm{Sec}$ & $2 \mathrm{Lh}^{-1}$ \\
\hline $4 \mathrm{Lh}^{-1}$ & $2 \mathrm{Lh}^{-1}$ & $4 \mathrm{Lh}^{-1}$ & $\mathrm{Sec}$ \\
\hline $2 \mathrm{Lh}^{-1}$ & $\mathrm{Sec}$ & $2 \mathrm{Lh}^{-1}$ & $4 \mathrm{Lh}^{-1}$ \\
\hline
\end{tabular}

\section{Verger expérinental: traitenents d'irrigation}

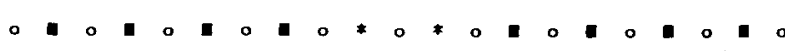

- Arbres de garde * Arbres étudiés o Goutteurs réels ou fictifs Placette élégentaire expérinentale

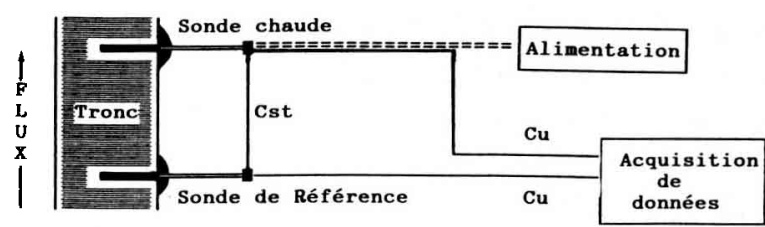

Fig 1. Verger expérimental et dispositif de mesure du flux de sève. 
Les arbres test retenus pour les mesures sont ceux qui, dans chaque traitement, présentent les caractéristiques morphologiques et végétatives moyennes des arbres du traitement considéré. Les flux horaires et journaliers sont respectivement donnés avec une incertitude relative inférieure à $15 \%$ et $10 \%$ (Cabibel. et Do, 1991).

\section{RÉSULTATS}

Ils concernent l'analyse des flux obtenus dans les troncs et les racines des arbres irrigués et non irrigués. Deux périodes de mesure sont considérées : la période de pré-irrigation (1-10 juillet 1986) et la période d'irrigation (12 juillet-11 août 1986), le déclenchement de l'apport d'eau au verger, étant effectué le 11 juillet. Les résultats présentés sont caractéristiques de chaque période analysée et des comportements moyens des arbres dans chaque traitement.

\section{Expressions des flux}

Ils sont exprimés soit :

- en densité de flux : exprimées en $\mathrm{ml}^{\mathrm{h}} \mathrm{h}^{-1} . \mathrm{cm}^{-2}$;

- en flux instantanés : exprimés en $\mathrm{ml}^{-h^{-1}}$;

- en flux cumulés : exprimés en mm.j-1. Compte tenu de la maille de plantation du verger, une surface, au sol, de $3,5 \mathrm{~m}^{2}$ est attribuée à chaque arbre, pour exprimer les flux en $\mathrm{mm}$.

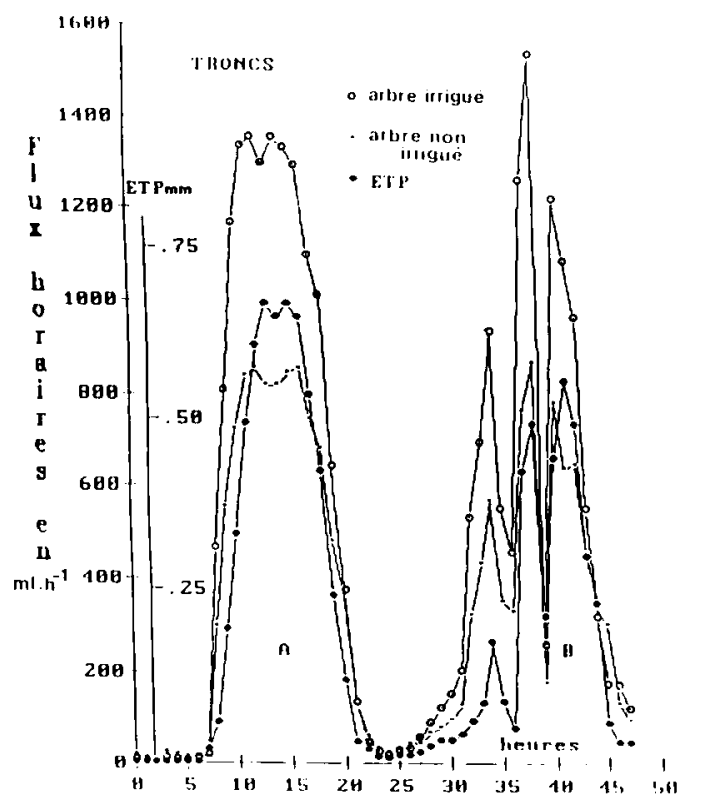

\section{Analyse des résultats}

\section{Cinétiques des flux de sève instantanés}

\section{Période de pré-irrigation}

Les variations nycthémérales de flux sont identiques dans les troncs et les racines des arbres des 2 traitements considérés (fig 2). Elles ne sont gouvernées que par la demande climatique.

\section{Par temps clair et ensoleillé}

Elles sont essentiellement constituées de 3 phases caractéristiques (fig $2 \mathrm{~A}$ ) :

- une phase de variation rapide des flux, consécutive à l'apparition et à la croissance de la demande climatique ( $8 \mathrm{~h}-10 \mathrm{~h})$,

- une phase de flux élevés variant plus lentement durant toute la période des fortes ETP $(11 \mathrm{~h}-16 \mathrm{~h})$,

- une phase de décroissance des transferts synchrone de la diminution de la demande climatique $(17 \mathrm{~h}-21 \mathrm{~h})$. L'annulation de cette dernière n'induit pas systématiquement l'absence d'un flux de sève dans l'arbre, notamment lors d'une séquence de fortes ETP journalières. Dans l'hypothèse d'un transfert dans l'arbre en flux non conservatif, ces flux nocturnes peuvent trouver leur origine dans une reconstitution des réserves en eau des parties aériennes de l'arbre, réserves ayant dans la phase initiale d'évapotran-

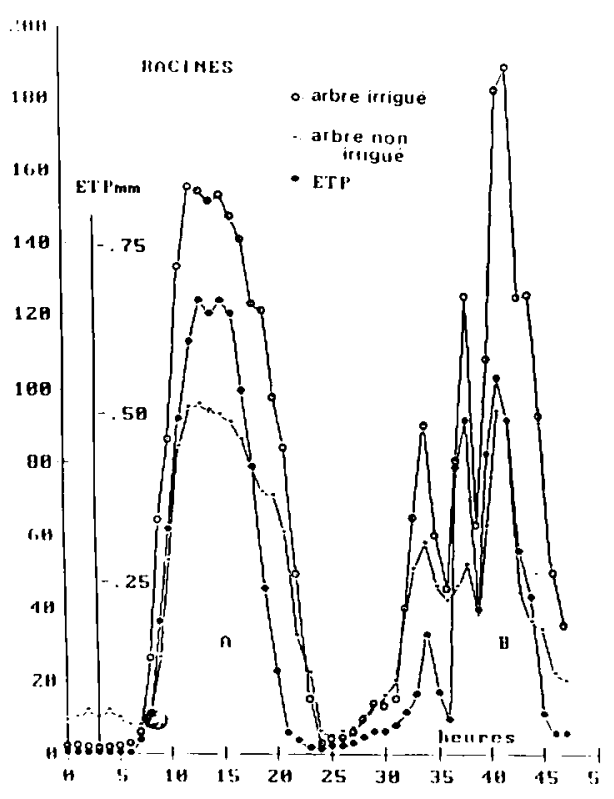

Fig 2. Cinétiques des flux horaires dans les troncs et les racines avant irrigation. 
spiration, participé à la satisfaction de la demande climatique (Huguet, 1985).

\section{Par temps nuageux}

Les cinétiques de flux sont strictement synchrones de l'évolution de la demande climatique. À toute élévation ou diminution de l'ETP correspond instantanément un accroissement ou une réduction des flux de sève (fig $2 \mathrm{~B}$ ).

\section{Période d'irrigation}

Pour une séquence climatique donnée, les cinétiques de flux sont étroitement liées aux traitements appliqués. Les flux dépendent donc de l'évolution relative de la disponibilité de l'eau du sol à l'interface sol-racine :

\section{En l'absence d'irrigation}

L'évolution des flux horaires, identique dans les troncs et les racines, est non synchrone de la demande climatique (fig 3 ). Si les flux nocturnes, peu élevés ou nuls, subissent encore une accélération importante à l'origine de celle-ci, les maxima de flux apparaissent, dans le temps, très antérieurement aux maxima de l'ETP. Ces flux maximaux ne se développent, de plus, que sur des temps courts. Ils décroissent en effet très rapidement, indépendamment de la très forte croissance existante de la contrainte climatique, et jusqu'à disparition de celle-ci.

L'analyse montre, de plus, que les maxima de flux apparaissent d'autant plus tôt, dans le temps, par rapport au maxima ETP que la période de non irrigation est longue, donc que la

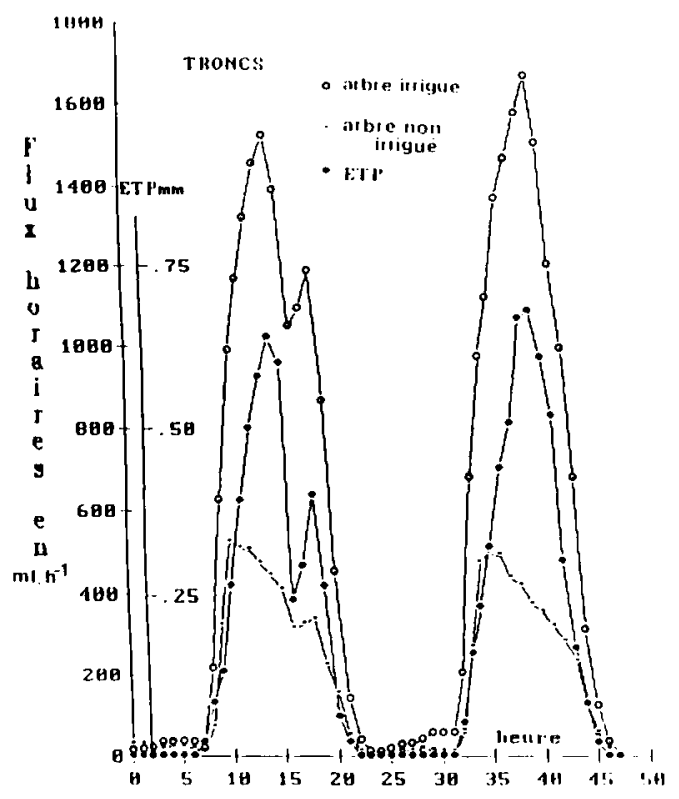

disponibilité de l'eau du sol diminue. On donne, en figure 4,3 cinétiques de flux caractérisant l'évolution de la fonction puits racinaire d'une même racine d'un pommier non irrigué. Elles ont été obtenues en période de pré-irrigation puis 24 et $28 \mathrm{j}$ après la date de déclenchement de l'apport d'eau sur les placettes irriguées. Aux 3 j considérés, les $E T P$ journalières étaient respectivement de $5,7 \mathrm{~mm}, 5,3 \mathrm{~mm}$ et $4,7 \mathrm{~mm}$, l'ETP horaire maximale étant identique et égale à 0,68 $\mathrm{mm}$. Aux 2 dernières dates étudiées, les déficits climatiques étaient, par rapport au jour de préirrigation considéré, de 200 et $220 \mathrm{~mm}$. L'étude des cinétiques de flux montre que, comme s'accroît l'assèchement du sol, apparaît, par rapport à l'ETP instantanée, un décrochage de plus en plus précoce et de plus en plus prononcé du flux de sève. Ce phénomène caractérise également le flux global mesuré dans les troncs.

\section{En présence d'irrigation}

Les évolutions des flux mesurées dans les troncs sont identiques à celles qui sont observées en période de préirrigation. Elles restent étroitement synchrones de la demande climatique (fig 3 ).

Dans les racines, les cinétiques de flux sont fonction de la localisation relative, dans le sol, de la zone d'absorption pour l'eau de la racine considérée et du volume mouillé par irrigation. Elles dépendent donc de la disponibilité relative locale de l'eau du sol, qui est elle-même fonction des conditions de mise-en-œuvre de l'irrigation. De ces dernières dépendent en effet les conditions de transfert dans le sol de l'eau apportée,

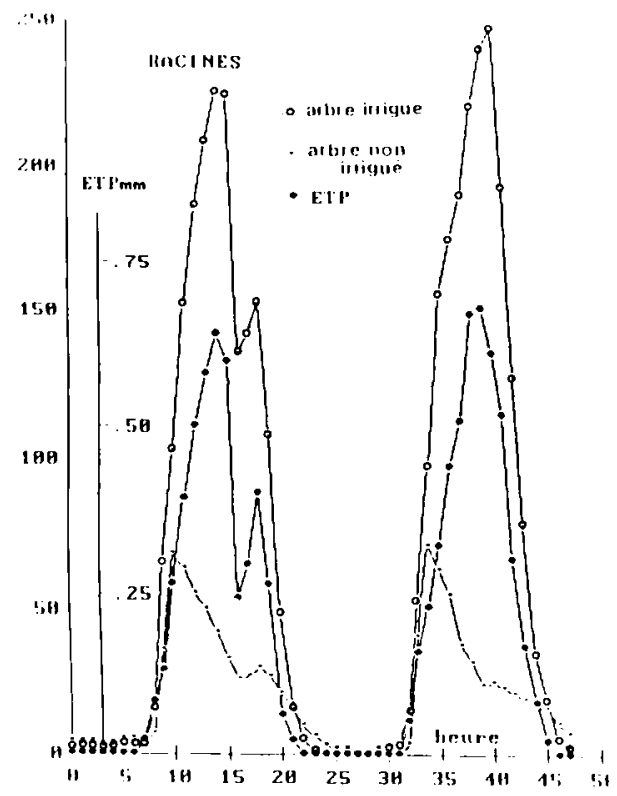

Fig 3. Cinétiques des flux horaires dans les troncs et les racines sous irrigation. 


\section{Conclusion partielle}

Lorsque la disponibilité de l'eau dans le sol n'est pas un facteur limitant de l'alimentation hydrique des arbres, les cinétiques nycthémérales des flux de sève ne sont gouvernées que par l'évolution de la contrainte climatique subie par le verger. Elles sont synchrones de celle-ci. En l'absence d'une telle disponibilité, les cinétiques de flux, non synchrones de l'ETP horaire, présentent une allure tronquée qui peut être considérée comme témoin d'un stress hydrique ou d'une régulation stomatique traduisant un besoin d'irrigation. L'analyse des flux de sève dans les racines individualisées permet d'accéder à l'étude de l'hétérogénéité spatiale et temporelle de la fonction puits racinaire des arbres.

L'étude des flux racinaires élémentaires doit donc permettre d'étudier les conditions de transfert dans le sol de l'eau apportée sous irrigation, notamment les temps de transfert du point d'apport en surface du sol aux sites d'extraction racinaire et la répartition des champs de flux et de pression. Elle doit, de plus, permettre d'analyser l'influence des diverses modalités de mise-enœuvre de l'apport d'eau en verger sur ces mêmes conditions de transfert dans le sol, sur l'efficience résultante de l'eau apportée, notamment sous irrigation localisée et sur la satisfaction des besoins en eau des arbres.

\section{Amplitude des flux}

\section{Période de préirrigation}

\section{Dans les troncs}

Les flux sont toujours plus élevés, à surfaces conductrices équivalentes, dans les arbres du traitement «irrigué». Ces différences doivent trouver leur origine dans l'existence de surfaces foliaires plus développées pour les arbres irrigués depuis leur plantation, du fait d'un plus grand développement. Une mesure de l'allongement des rameaux, faite sur 4 années successives, montre en effet, durant cette période, une croissance systématiquement supérieure de $50 \%$ en faveur des rameaux de l'année des arbres de ce traitement. Les flux sont donc fonction du passé cultural de l'arbre. Les rapports des flux moyens instantanés (fig 2A), mesurés pour les $\mathrm{h}$ de plus forte demande climatique (12 h-16 h) et respectivement égaux, aux $2 \mathrm{j}$ considérés dans les 2 traitements, à 1365 et $968 \mathrm{ml}^{-\mathrm{h}^{-1}}$ (irrigué) et à 835 et $598 \mathrm{ml}^{-\mathrm{h}^{-1}}$ (sec) sont voisins de 1,60. Les rapports des flux jour- naliers correspondants, respectivement égaux à 3,93 et 3,28 (irrigué) et à 2,61 et $2,19 \mathrm{~mm}$ (sec), sont voisins de 1,50.

\section{Dans les racines}

Les flux apparaissent, en valeur brute, également plus élevés dans les racines du traitement irrigué. Néanmoins, les racines testées ayant, contrairement aux troncs, des surfaces conductrices différentes, $14,25 \mathrm{~cm}^{2}$ (Irrigué) et $4,21 \mathrm{~cm}^{2}$ (Sec), seule l'analyse des vitesses de flux peut permettre une approche comparative réelle de l'intensité des flux (fig 6). Leur analyse montre que l'activité racinaire n'a pas de relation directe avec le traitement. Elle doit être seulement reliée à la disponibilité relative locale de l'eau du sol à l'interface sol-racine. Dans le cas présenté, le rapport moyen horaire des densités de flux est égal à 2,0 en faveur des racines du traitement "sec". En conséquence dans un sol où la répartition de l'eau est théoriquement homogène, il apparaît :

- soit que la racine de l'arbre non irrigué est "plus active" dans l'extraction de l'eau du sol (exploration d'horizons plus profonds et/ou maillage plus fin des sites d'absorption par adaptation du système racinaire en condition de non irrigation),

- soit que la fonction puits racinaire et l'évaporation de surface déjà réalisées, préalablement à la période d'étude, aient induit une hétérogénéité dans la répartition spatiale des réserves initiales en eau du sol, ayant des conséquences sur le fonctionnement racinaire analysé, les 2 racines étudiées pouvant explorer des horizons différents.

Pour une contrainte horaire donnée, l'intensité du flux horaire correspondant est fonction du type de séquence des contraintes climatiques horaires s'exerçant sur le verger (fig 2) :

- dans une séquence régulière des contraintes climatiques horaires (jour clair et ensoleillé), l'intensité des flux horaires est fonction, quel que soit l'organe testé, de l'intensité de la contrainte climatique subie (fig 2). Néanmoins si les flux de sève croissent avec la demande climatique horaire, leur intensité est d'autant moins proportionnelle à l'ETP que celle-ci devient grande. Les flux se stabilisent à un maximum ou tendent à diminuer dès que la contrainte climatique dépasse un seuil donné. lls décroissent ensuite linéairement avec l'ETP, après passage au maximum de cette variable, sans pour cela s'annuler systématiquement avec elle. 

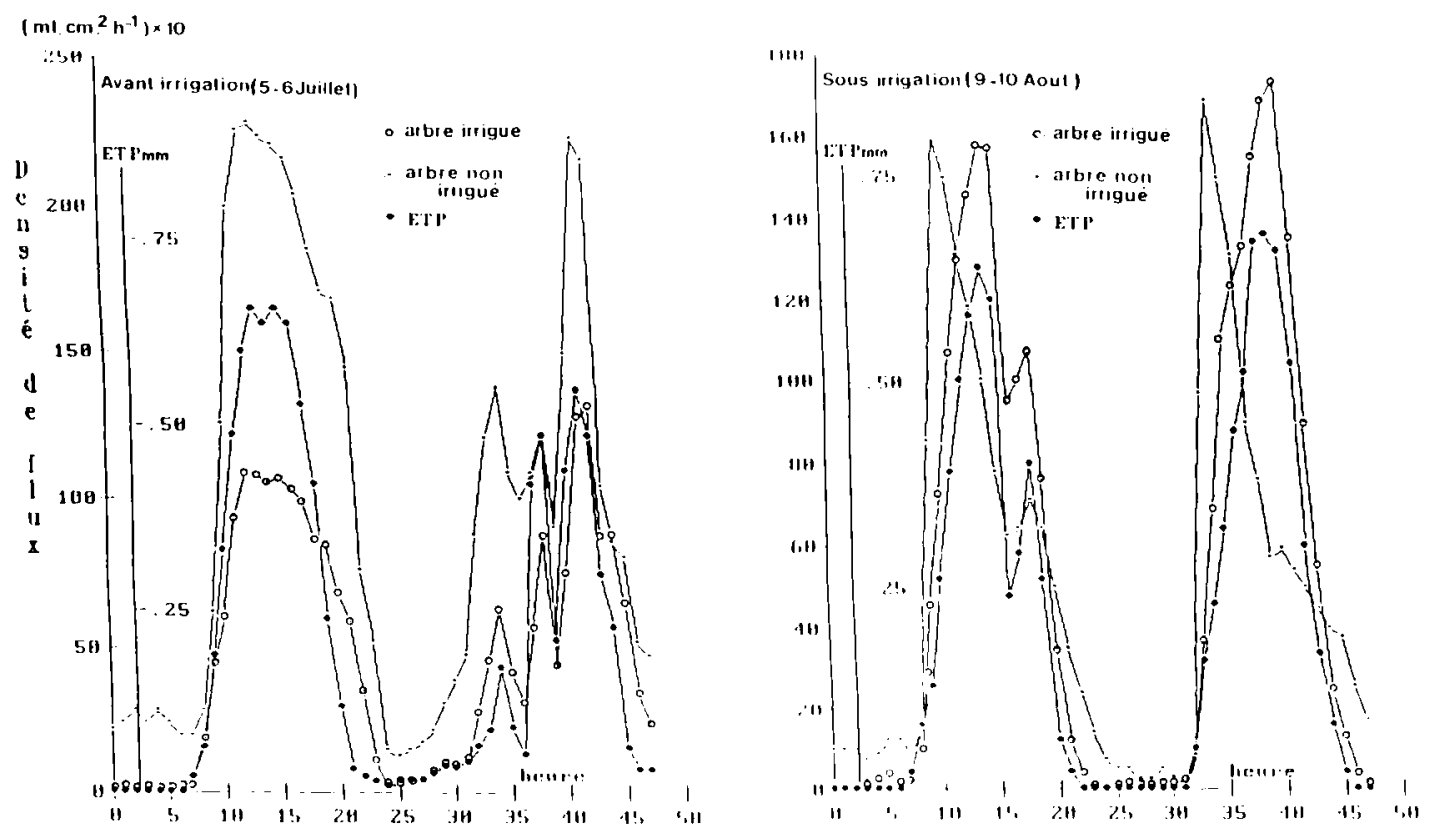

Fig 6. Cinétiques des densités de flux racinaires avant et durant la période d'irrigation.

Par ailleurs, les flux mesurés dans les troncs sont toujours, pour une ETP horaire donnée, plus forts en phase de croissance de la contrainte climatique que dans la phase de décroissance de cette variable. Inversement, dans les racines, les flux sont plus élevés en phase de décroissance des ETP horaires. Dans l'hypothèse d'une hiérarchisation dans l'utilisation et la reconstitution des diverses réserves en eau du végétal, ayant participé à la satisfaction de l'ETP lors de sa phase initiale, l'importance des flux racinaires tardifs pourrait signifier, in fine, une reconstitution active des réserves préalablement mobilisées dans ces organes (Garnier, 1985).

Dans une séquence irrégulière des contraintes climatiques (jour avec passage nuageux) l'intensité du flux, pour une ETP horaire donnée, est aussi fonction de l'intensité des ETP horaires précédentes. Les rapports flux/ETP sont toujours plus forts, à contrainte climatique égale, lorsque cette contrainte a été précédée d'une diminution de la demande climatique (fig 2B). Ce phénomène pourrait être sous la dépendance de l'évolution du degré de disponibilité de l'eau à l'interface sol-racine. Sous une demande élevée et continue, la conductivité hydraulique faible du sol ne permettrait pas le maintien à un niveau élevé de la disponibilité de l'eau à l'interface sol-racine. Il en résulterait une diminution de l'absorption racinaire et donc des flux. Inversement toute diminution momentanée de la demande climatique permettrait la restauration d'une disponibilité accrue en eau à l'interface sol-racine entra prélèvement supérieur pour un gradient ETP donné à venir. Il en est de même des flux cumulés mesurés si, exprimés en \% de l'ETP totale. Ils sont proportionnellement plus élevés lors d'une séquence non régulière d'ETP horaires. Pour les 2 j présentés, un jour régulièrement ensoleillé et un jour nuageux, ils sont respectivement égaux, dans les 2 traitements, à $69 \%$ et $45 \%$ de l'ETP le premier jour et à $85 \%$ et $56 \%$ de l'ETP le second (fig 7).

\section{Période d'irrigation}

Pour une ETP donnée, l'intensité des flux diminue de façon continue comme diminue la teneur en eau du sol en l'absence d'irrigation. Inversement, en présence des apports d'eau journaliers, elle se maintient à des niveaux élevés ou augmente, les réserves en eau mobilisables étant maintenues maximales dans la zone d'activité racinaire (figs 3,6 et 7 ). On donne, tableau I, des valeurs de flux instantanés et cumulés, représentatives des phénomènes étudiés dans les 2 traitements lors des 2 périodes considérées. Leur analyse montre que si la fonction puits racinaire instantanée totale satisfait, sous irrigation, la totalité de la demande climatique aux faibles valeurs de celle-ci, le flux n'excède jamais, en moyenne, plus de $75 \%$ de l'ETP dès que celle-ci devient supérieure de $0,5 \mathrm{~mm} . \mathrm{h}^{-1}$, la valeur de satisfaction diminuant comme augmente l'amplitude de la contrainte. Les flux cumulés représentent au maximum $85 \%$ de l'ETP journalière sous 

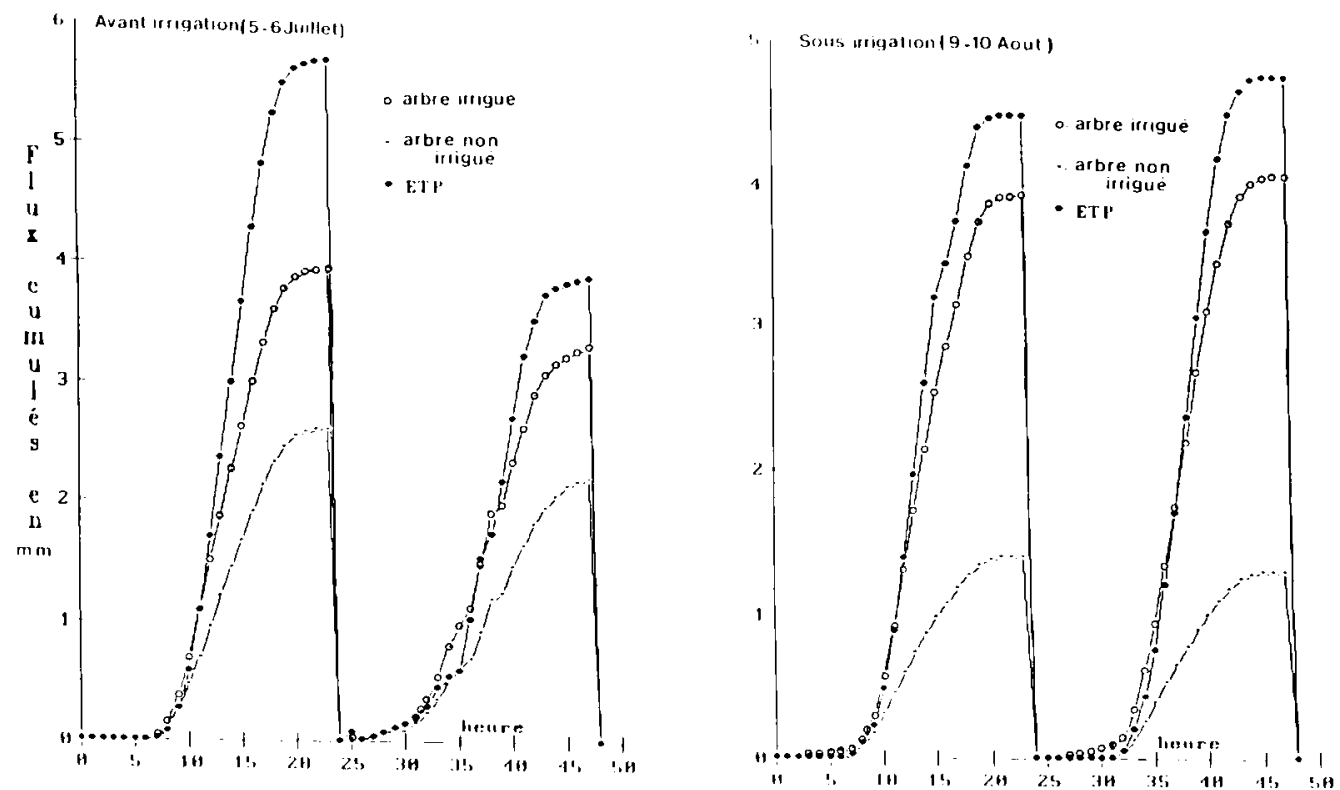

Fig 7. Flux cumulés dans les arbres avant et durant la période d'irrigation.

irrigation, même en présence de dose d'eau supérieure à l'ETP (cas expérimenté). Ce résultat est à rapprocher du fait que les arbres, de par leur structure de plantation et leur mode de conduite, n'interceptent pas la totalité du rayonnement solaire arrivant sur le verger. En traitement «sec» ils peuvent atteindre $30 \%$ de l'ETP.
L'évolution des valeurs de flux est identique dans les racines. Pour une même contrainte de $0,64 \mathrm{~mm} \cdot \mathrm{h}^{-1}$, la racine du traitement irrigué, présente dans le bulbe humide, voit son activité s'élever de $50 \%$ en phase d'irrigation, alors que celle du traitement sec diminue corrélativement de $54 \%$ (tableau I).

Tableau I. Évolution des flux mesurés en période de pré-irrigation et d'irrigation, selon les traitements.

\begin{tabular}{|c|c|c|c|c|c|c|c|c|c|}
\hline \multirow[t]{2}{*}{$E T P$} & \multicolumn{4}{|c|}{ Traitement } & \multicolumn{5}{|c|}{ Rapports } \\
\hline & & & & & & & & & NI/ETP \\
\hline$P I^{\star}$ & $P I$ & 1 & $P I$ & 1 & $P I$ & 1 & $P I$ & 1 & $P I$ \\
\hline
\end{tabular}

Flux instantané

(mI. $\left.h^{-1}\right)$

pour $E T P=0,64 m m \cdot h^{-1}$

Tronc

Racine

$\begin{array}{rrlrrr}1350 & 1518 & 816 & 441 & 1,654 & 3,440 \\ 151 & 225 & 91 & 42 & 1,659 & 5,357\end{array}$

Densité de flux

(ml. $\mathrm{h}^{-1} . \mathrm{cm}^{-2}$ )

pour ETP $=0,64 m m \cdot h^{-1}$

\begin{tabular}{|c|c|c|c|c|c|c|c|c|c|c|c|}
\hline Racine & & 106 & 158 & 223 & 100 & 0,475 & 1,580 & & & & \\
\hline $\begin{array}{l}\text { Flux cumulé } \\
\left(m m \cdot \dot{j}^{-1}\right)\end{array}$ & 5,7 & 3,93 & 4,07 & 2,61 & 1,29 & 1,506 & 3,155 & 0,689 & 0,848 & 0,457 & 0,269 \\
\hline Rapport I/PI & {$[0,842]$} & {$[1,0$} & & {$[0,4$} & & {$[2$,} & & & 232] & {$[0,5$} & 89] \\
\hline
\end{tabular}

$\mathrm{I}$ : valeur de la variable en période irriguée; $\mathrm{NI}$ : valeur de la variable en période non irriguée; $\mathrm{PI}$ : valeur de la variable en période de pré-irrigation. 


\section{Conclusion partielle}

En phase de pré-irrigation les amplitudes instantanées et cumulées des flux dépendent, dans les troncs, de l'intensité de la demande climatique, et pour une contrainte climatique donnée, du passé cultural de l'arbre qui a commandé son développement. Dans les racines, elles dépendent aussi de la disponibilité locale de l'eau dans le sol. Les flux sont également fonction de type de séquence des contraintes climatiques horaires s'exerçant sur le système sol-plante. Ils dépendent donc du type de séquences des équilibres instantanés successifs existant entre l'intensité de la demande climatique et l'aptitude du sol à maintenir une disponibilité élevée de l'eau à l'interface sol-racine. En phase d'irrigation l'intensité des flux est essentiellement sous la dépendance de la mobilité de l'eau dans le sol et de son évolution dans la zone d'activité racinaire.

\section{CONCLUSIONS}

La quantification, par voie thermique des cinétiques de flux de sève, dans les troncs et les racines d'arbres fruitiers permet:

- d'appréhender le comportement hydrique relatif de ces arbres et leurs évolutions, sous les différentes formes que sont

- le transfert de sève à l'échelle horaire, tant globale au niveau des troncs que dans ses composantes au niveau des racines,

- le transfert de sève à l'échelle journalière. Celui-ci pourrait être, par ailleurs, considéré comme une estimation de la transpiration au jour considéré si la mesure couplée du potentiel de base dans l'organe étudié montrait une égalité de ce potentiel au jour $n-1$ et au jour $n$, la fraction d'eau mobilisée par la croissance devant être négligeable devant la transpiration,

- la reconstitution probable des réserves en eau initiales propres à l'arbre, réserves ayant participé à la phase initiale d'évapotranspiration, au travers de flux racinaires tardifs mis en évidence en l'absence de toute contrainte climatique;

- de différencier les comportements hydriques des arbres dans les différentes périodes de culture, pré-irrigation et irrigation. Elle permet notamment de caractériser, au travers de cinétiques de flux mettant en évidence des décalages importants, dans le temps, entre les maxima de flux et de la contrainte climatique s'exerçant sur le verger, l'apparition probable d'un stress hydrique dans les arbres et donc le besoin d'irrigation;

- d'appréhender et de quantifier l'hétérogénéité spatio-temporelle de la fonction puits racinaires de l'arbre liée à l'hétérogénéité naturelle ou induite de la disponibilité de l'eau du sol.

L'analyse des flux de sève dans les différentes composantes de l'arbre, paramètre traduisant, à un pas de temps court, l'extraction de l'eau du sol par les racines et son évolution comme résultant des équilibres instantanés successifs entre la disponibilité de l'eau du sol et l'intensité de la demande climatique, doit donc permettre :

- d'optimiser l'origine dans le temps et le débit de l'apport d'eau,

- de juger, pour des conditions d'irrigation localisée et de structure de sol données mais variables

- des localisations relatives de la zone mouillée par rapport à celle du système racinaire actif,

- des interrelations existantes aux interfaces solracine, quant à la disponibilité de l'eau d'irrigation et de son prélèvement par les racines,

- de juger de l'efficacité de l'apport d'eau et des modalités de mise en œuvre de l'irrigation, au travers du degré de satisfaction des besoins en eau des arbres,

- d'envisager une méthode de pilotage de l'irrigation comme prolongement finalisé de ces mesures, notamment si ces mesures de flux sont couplées à des mesures micrométriques des variations des diamètres des organes de l'arbre et à celles des potentiels de base dans la plante.

\section{RÉFÉRENCES}

Cabibel B, Do F (1990) Mesures thermiques des flux de sève dans les troncs et les racines et fonctionnement hydrique des arbres. I. Analyse théorique des erreurs sur la mesure des flux des mesures en présence de gradients thermiques extérieurs. Agronomie 11, 669-678

Cohen Y, Fuchs G, Green GC (1981) Improvement of the heat pulse method for determining sap flow in trees. Plant Cell Environ 4, 391-397

Do $F(1986)$ Estimation des flux racinaires et du comportement hydrique du pommier sous irrigation lo- 
calisée en sol fissuré. Mémoire de fin d'études, ENIT, Bordeaux

Garnier E (1985) Dessèchement du sol et indicateurs physiologiques de l'état hydrique chez le pêcher : application au déclenchement de l'irrigation. Thèse Docteur-Ingénieur en agronomie. ENSAM Montpellier

Granier A (1985) Une nouvelle méthode pour la mesure des flux dans le tronc des arbres. Ann Sci For $45,193-200$
Huguet JG (1985) Appréciation de l'état hydrique d'une plante à partir des variations micrométriques de la dimension des fruits ou des tiges au cours de la journée. Agronomie 5, 733-742

Pinguet A (1984) Utilisation de la station météoautomatique Cimel à 5 capteurs. Note interne, INRA

Valancogne C, Nasr $Z$ (1989) Une méthode de mesure du débit de sève brute dans de petits arbres par bilan de chaleur. Agronomie 9, 609-617 